Retraction

\title{
Retracted: Combined Location-Inventory Optimization of Deteriorating Products Supply Chain Based on CQMIP under Stochastic Environment
}

\author{
Mathematical Problems in Engineering \\ Received 5 March 2019; Accepted 5 March 2019; Published 7 April 2019 \\ Copyright (c) 2019 Mathematical Problems in Engineering. This is an open access article distributed under the Creative Commons \\ Attribution License, which permits unrestricted use, distribution, and reproduction in any medium, provided the original work is \\ properly cited.
}

Mathematical Problems in Engineering has retracted the article titled "Combined Location-Inventory Optimization of Deteriorating Products Supply Chain Based on CQMIP under Stochastic Environment" published in Mathematical Problems in Engineering in 2018 [1]. The article was found to contain significant material from another article titled "A Conic Integer Programming Approach to Stochastic Joint Location-Inventory Problems" published in Operations Research in 2012 by different authors [2], which was not cited.

Several parts are copied word by word from the other article [2]. In addition, Figure 2 in [1] is similar to Figure 1 in [2], while the results in Figures 3, 4, and 5 in [1] appear to be the same as Figures 2, 3, and 4, respectively, in [2]. Moreover, there are close similarities in the equations and tables. Finally, the results in the Table 1 in [1] and Table 3 in [2] are very closely correlated, despite supposedly not using the same model.

The Mathematical Problems in Engineering authors clarified that their article is different from the other one as they designed a combined location-inventory model for deteriorating products under capacitated facilities, stochastic lead time, multiple products, and correlated retailers' stochastic demands assumptions. Mathematical Problems in Engineering asked the authors to provide evidence that they conducted these simulations, e.g., code and raw outputs. The authors said the data are already included in the article and no additional data can be provided, and they provided code.

The Editorial Board found that the code and equations provided by the authors did not help determine whether the results are original. Accordingly, we asked their institutions to formally investigate.

\section{References}

[1] Y. M. Wang and H. L. Yin, "Combined location-inventory optimization of deteriorating products supply chain based on CQMIP under stochastic environment," Mathematical Problems in Engineering, vol. 2018, Article ID 8210254, 10 pages, 2018.

[2] A. Atamtürk, G. Berenguer, and Z.-J. Shen, "A conic integer programming approach to stochastic joint location-inventory problems," Operations Research, vol. 60, no. 2, pp. 366-381, 2012. 


\title{
Combined Location-Inventory Optimization of
} Deteriorating Products Supply Chain Based on CQMIP under Stochastic Environment

\author{
Yong Ming Wang $\mathbb{D}^{1}$ and Hong Li Yin $\mathbb{D}^{2}$ \\ ${ }^{1}$ Faculty of Management and Economics, Kunming University of Science \& Technology, Kunming 650093, China \\ ${ }^{2}$ School of Computer Science and Information Technology, Yunnan Normal University, Kunming 650500, China
}

Correspondence should be addressed to Hong Li Yin; hongli_yin@163.com

Received 2 September 2017; Revised 13 December 2017; Accepted 3 January 2018; Published 5 February 2018

Academic Editor: Mauro Gaggero

Copyright (c) 2018 Yong Ming Wang and Hong Li Yin. This is an open access article distributed under the Creative Commons Attribution License, which permits unrestricted use, distribution, and reproduction in any medium, provided the original work is properly cited.

\begin{abstract}
The design and optimization of combined location-inventory model for deteriorating products are a main focus in supply chain management. There were many combined location-inventory design models in this field, but these models are under the assumptions of adequate capacity facilities, invariable lead time, unique product, and uncorrelated retailer's demands. These assumptions have a big gap in the practical situation. In this paper, we design a combined location-inventory model for deteriorating products under capacitated facilities, stochastic lead time, multiple products, and correlated retailers' stochastic demands assumptions. These constraints are near to actual supply chain circumstance. The problem is modeled as conic quadratic mix-integer programming (CQMIP) to minimize the total expected cost. We explain how to formulate these problems as conic quadratic mixed-integer problems, and in order to obtain better computational results we use extended cover cuts. Simultaneously we compare our method with the previous Lagrange methods; the result is that the new CQMIP method can get better solution.
\end{abstract}

\section{Introduction}

Design and optimization of the combined location-inventory for deteriorating products are extremely important. Deterioration is defined as "the damage, spoilage, dryness, etc. that results in a decrease of usefulness of the original one" [1]. Deteriorating products are classified as two kinds. The products that have a maximum usable lifetime are seen as "perishable products," like foodstuffs, green vegetables, human blood, and photographic film. And the products that have no shelf-life at all are seen as "decaying products," like alcohol, gasoline, and radioactive substances [2].

Deteriorating products about a third of sales at retailers, combined location-inventory design and optimization of the deteriorating products become a attention point. These problems are more difficult than combined location-inventory design and optimization of products with an infinite lifetime. We will consider minimizing the total expected cost under satisfying customer demand. In many cases, the holding cost and transportation cost per unit or per unit time for deteriorating products are not fixed. The cost of each unit deteriorating product is alterable; it depends on time, inventory level, storage location, and so forth. Therefore, the combined location-inventory system's costs of deteriorating products are more complex. The influence of deteriorating products on the environment is a real and big problem; more and more academic researchers are paying attention to these deteriorating products supply chain.

There are numerous challenges and opportunities for deteriorating products supply chain. However, few rigorous and general supply chain models have been developed. Therefore, it is worthwhile and necessary to research deteriorating products supply chain. There are impacts on the economy and positive effects on health. At present, few works consider combined location-inventory in deteriorating products supply chain design and optimization. The majority of studies on deteriorating products are limited to inventory 
level or location level, rarely involved in combined locationinventory aspect. Hence, this paper considers a combined deteriorating supply chain design and optimization problem; we design a combined location-inventory model for deteriorating products. In our model, in order to make the model more realistic we consider capacitated facilities, stochastic lead time, multiple products, and correlated retailer's demands.

In this paper, we propose a novel method based on conic quadratic mixed-integer programming, which is more flexible and global. We use optimization software CPLEX to solve this problem. The experimental results show that, compared to Lagrangian relaxation, however, our method has many advantages. And this research work provides an effective reference for managers of deteriorating products supply chain.

The remainder of this paper is organized as follows. In Section 2, we review the main researches in combined location-inventory models of supply chain. The notation and modeling assumptions are described in Section 3. In Section 4, a CQMIP model is presented for the combined location-inventory of deteriorating products. Numerical results together with related analyzes are presented in Section 5 . Section 6 concludes this paper and briefly discusses future research directions.

\section{Literature Review}

Supply chain design and optimization are critical and difficult. It involves not only facility location but also facility inventory. Most of existing supply chain design and optimization models separately consider location decision and inventory decision, with no regard for the interaction between decision of location and decision of inventory. In order to reach efficient cost savings, the major cost components of location and inventory should be considered in combination, rather than separately. Thus, the idea of considering inventory costs while making facility location decisions of a supply chain has been developed during the past decade by different researchers. These models concurrently consider decisions at both the strategic location decisions level and the tactical inventory decisions level. Snyder et al. [3] proposed a stochastic location-inventory model with uncertainty. Shen and Qi [4] proposed a combined location-inventory routing decisions model. Ozsen et al. [5], directed at limited capacities for facilities, proposed an integrated location-inventory model. In these works, because of inventory consideration, the objective function is nonlinear. These models were complex, usually with integer decision variables, nonlinear costs, and constraints. So they proposed many heuristic algorithms for different special cases. Shen et al. [6] proposed a column generation method for this problem. Daskin et al. [7] and Shen and Daskin [8] all used Lagrangian relaxation method to solve this class of problems. Miranda and Garrido [9] considered facility location and inventory control decisions and presented an integrated model; the solution method involves Lagrangian relaxation and subgradient method. Erlebacher and Meller [10] proposed a two-stage heuristic algorithm to estimate the number of DCs. Dasci and Verter
[11] introduced concave technology selection cost into the objective function of a multiproduct location model. AslNajafi et al. [12] considered a dynamic closed-loop locationinventory problem and designed a hybrid metaheuristic algorithm based on multiobjective particle swarm optimization (MOPSO) and nondominated sorting genetic algorithm-II (NSGA-II) to solve the problem. Zhang and Unnikrishnan [13] presented a location-inventory model with uncertain demands, which is based on integer nonlinear programming and can be transformed to conic quadratic mixed-integer programming, and used CPLEX software solve this problem. Diabat et al. [14] presented a joint location-inventory model based on uncertain demands and lead times, the use of which can determine not only the location and number of distribution centers but also the size, and adopted a hybrid algorithm to solve the presented model; this algorithm arose from simulated annealing and direct search. Vahdani et al. [15] considered a model based on mixed-integer nonlinear programming for a location-inventory problem, which assumed that the demands of retailers are correlated, and presented two metaheuristic algorithms, including genetic algorithm and simulated annealing to solve this problem.

Because the feature of the deteriorating products, there is a strict time restrict about deteriorating products. The product will lose its value rapidly, which can lead to extra waste and costs and loss potential market share. With increasing time-sensitivity for deteriorating products, timely delivery is becoming a strategy. A great number of tactics for time competition have been proposed; several methods were utilized for time-dependent supply chain design and optimization. Rich and Hines [16] proposed mathematical modeling, which focuses on time reduction for a single specific process. Guide Jr. et al. [17] discussed profit maximization in reverse supply chains for deteriorating return. Blackburn and Scudder [18] developed a cost minimization model for one specific deteriorating product supply chain, concerning the declining value of the product over time. Wang et al. [19] discussed inventory policies for a deteriorating product but based on a simple supply chain system consisting of one producer, one distributor, and only one retailer. Arntzen et al. [20] proposed mixed-integer linear programming model to minimize the weighted combination of total cost and activity days, but the unit processing activity days are fixed, with no regard for the facility capacities.

In the existing literature on deteriorating products, for describing either the decrease in quantity or the degeneration in quality, exponential time decay has been utilized. Regarding the decrease in quantity, Nahmias [21] and Yu and Nagurney [22] discussed perishable inventory optimization. Tijskens and Polderdijk [23] and Akkerman et al. [24] discussed the degeneration in quality but assumed that all the products deteriorate at the same rate.

\section{Model Assumptions and Notations}

The structure of the integrated supply chain is as in Figure 1. It is a three-level supply chain under the single-sourcing assumption; it has a central manufacturer, a set of distribution 


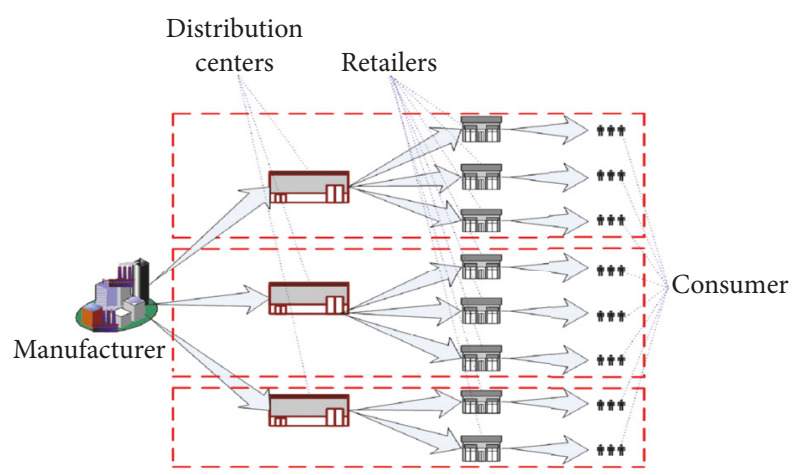

FIgURE 1: The three-level supply chain network.

centers (DCs) that store and distribute supplies, and the retailers.

We used parameters and notations for the CQMIP model as follows:

Sets

$L$ : set of deteriorating products, indexed by $l$

$I$ : set of retailers, indexed by $i$

$J$ : set of potential distribution center locations, indexed by $j$

Demands

$\mu_{i l}$ : the daily demand at retailer $i$ for deteriorating product $l$

$\delta_{i l}$ : standard deviation of daily demand at retailer $i$ for deteriorating product $l$

$V_{l}$ : variance-covariance matrix of retailers daily demand about deteriorating product $l$

Costs

$f_{j}$ : fixed cost of DC located at retailer site $j$ per unit time

$d_{i j l}$ : cost per unit to transport deteriorating product $l$ between site $i$ and $j$

$F_{j l}$ : fixed cost of placing an order deteriorating product $l$ at DC $j$

$F_{l}$ : fixed cost of placing an order deteriorating product $l$ at central manufacturer

$a_{j l}$ : per unit cost to transport deteriorating product $l$ from the central manufacturer to $\mathrm{DC} j$

$g_{j l}$ : fixed cost of per unit transport deteriorating product $l$ from the central manufacturer to $\mathrm{DC} j$

$h_{l}$ : unit inventory holding cost per unit of deteriorating product $l$ per unit time

$D_{l}$ : fixed disposing cost of deteriorating product $l$ after it becomes putrid

Weights

$\beta_{l}$ : weight factor associated with the transportation cost for the deteriorating product $l$

$\theta_{l}$ : weight factor associated with the inventory cost for the deteriorating product $l$

\section{Other Parameters}

$x_{l}$ : days worked per unit time for the deteriorating product $l$

$\alpha^{l}$ : service level of deteriorating product $l$

$z_{\alpha^{l}}$ : standard normal deviation associated with $\alpha^{l}$

$L_{j l}$ : lead time in days for deteriorating product $l$ at DC j

$\sigma_{L_{j l}}$ : standard deviation of lead time in days for deteriorating product $l$ at DC $j$

$t_{j}$ : time of transportation of deteriorating product from the central manufacturer to $\mathrm{DC} j$

$t_{i j}$ : time of transportation of deteriorating product from site $j$ to site $i$

$t_{l}$ : maximum usable lifetime of deteriorating product $l$

$c_{j}$ : maximum inventory capacity of DC $j$

$N_{l 0}$ : quantity of deteriorating product $l$ at DC $j$

$\lambda_{l}$ : decay rate of deteriorating product $l$

\section{Decision Variables}

$y_{i j l}=1$ : if demand for deteriorating product $l$ of retailer $i$ is assigned to DC at retailer site $j$

$y_{i j l}=0$ : if demand for deteriorating product $l$ of retailer $i$ is not assigned to DC at retailer site $j$

$x_{j}=1$ : if DC is located at retailer site $j$

$x_{j}=0$ : if DC is not located at retailer site $j$

$Q_{j l}$ : the reorder number of deteriorating product $l$ at DC $j$.

We also consider the following assumptions:

(1) Each retailer is supplied from unique DC.

(2) Each DC has nonlinear capacity constraints.

(3) Deteriorating products are transported directly from DCs to retailers.

(4) Objective function is nonlinear.

(5) Although the demand of retailer $i$ for deteriorating product $l$ is stochastic, there is a correlation between different retailers' demands.

(6) Lead times of deteriorating products are independent and orders not crossed.

(7) The lead time of deteriorating product is stochastic.

(8) Every deteriorating product has a lifetime; product value decreases over time.

(9) The environmental conditions associated with each deteriorating product are fixed, such as temperature and transportation. 


\section{CQMIP Model}

At least one of the variables is unknown in our model; we assume they follow some probability distribution. Stochastic supply chain optimization and design problems were usually described as integer or mixed-integer nonlinear optimization problems. In our paper, we will propose a combined locationinventory model based on conic quadratic mixed-integer programming. In this section, the conic quadratic mixedinteger programming (CQMIP) optimization problem is described first. Then, we use conic quadratic mixed-integer programming model of complex deteriorating product supply chain optimization and design problems.

4.1. CQMIP Optimization Problem. Now we refer to BenTal and Nemirovski [25] and Alizadeh and Goldfarb [26] who define conic quadratic mixed-integer programming (CQMIP) optimization problem: $\min a^{\prime} x$

$$
\text { s.t. } \quad\left\|A_{i} x+b_{i}\right\|_{2} \leq c_{i}^{\prime} x+d_{i}, \quad i=1, \ldots, p
$$

where $x \in Z^{n} \times R^{m},\|\cdot\|_{2}$ is the Euclidean norm, and all the parameters are rational numbers. Let $c^{\prime}=0, A_{i}=b_{i}=0$; we can obtain convex quadratic constraint.

4.2. CQMIP Model. In this section, we are going to consider the multiple deteriorating products combined locationinventory model with inventory capacity constraint, correlated retailers' stochastic demands, stochastic lead times, and maximum usable lifetime.

Based on the above assumptions, the multiple deteriorating products combined location-inventory model is indicated in problem $(P)$ as follows:

$$
\begin{array}{ll}
\min & \sum_{j \in J}\left(f_{j} x_{j}+\sum_{l \in L}\left(\sum_{i \in I} \hat{d}_{i j l} y_{i j l}+\hat{F}_{j l} \frac{\sum_{i \in I} \mu_{i l} y_{i j l}}{Q_{j l}}+\theta h_{l} \frac{Q_{j l}}{2}+\hat{q}_{j l} \sqrt{y_{. j l}^{\prime}\left(L_{j l} V_{l}+\sigma_{L_{j l}}^{2} M_{l}\right) y_{. j l}}+\sum_{i \in I} N_{i 0}\left(1-e^{-\lambda_{l}\left(t_{j}+t_{i j} y_{i j l}+L_{j l}\right)}\right)\left(F_{i}+D_{i}\right)\right)\right), \\
\text { s.t. } \quad & \sum_{l \in L}\left(L_{j l} \sum_{i \in I} \mu_{i l} y_{i j l}+Q_{j l}+z_{\alpha^{l}} k_{l} \sqrt{y_{. j l}^{\prime}\left(L_{j l} V_{l}+\sigma_{L_{j l}}^{2} M_{l}\right) y_{. j l}}\right) \leq C_{j} x_{j}, \quad j \in J, \\
& \left(t_{j}+L_{j l}+y_{i j l} t_{i j}\right) \leq t_{l}, \quad i \in I, \quad j \in J, l \in L \\
& \sum_{j \in J} y_{i j l}=1, \quad i \in I, l \in L \\
& y_{i j l} \leq x_{j}, \quad i \in I, \quad j \in J, l \in L, \\
& Q_{j l} \geq 0, \quad j \in J, l \in L,
\end{array}
$$

where $\hat{d}_{i j l}=\beta_{l} x_{l}\left(d_{i j l}+a_{j l}\right) \mu_{i l}$ is the cost of transportation product $l$ to retail site $i . \hat{F}_{l}=\left(F_{j l}+\beta_{l} g_{j l}\right) x_{l}, \hat{q}_{j l}=$ $z \alpha^{l} \theta_{l} \sqrt{L_{j l}} h_{l}, y_{. j l}^{\prime}=\left(\begin{array}{c}y_{1 j l} \\ \vdots \\ y_{I j l}\end{array}\right)$ is the assignment decision matrix for the $j$ th $\mathrm{DC}$ for the deteriorating product $l$. $V_{l}$ is variancecovariance matrix of daily demand at retailers to deteriorating product $l$. And

$$
M_{l}=\left(\begin{array}{cccc}
\mu_{1 l}^{2} & \mu_{1 l} \mu_{2 l} & \cdots & \mu_{1 l} \mu_{I l} \\
\mu_{2 l} \mu_{1 l} & \mu_{2 l}^{2} & \cdots & \mu_{2 l} \mu_{I l} \\
\vdots & \vdots & & \vdots \\
\mu_{I l} \mu_{1 l} & \mu_{I l} \mu_{2 l} & \cdots & \mu_{I l}^{2}
\end{array}\right)
$$

$e^{-\lambda_{l}\left(t_{j}+t_{i j} y_{i j l}+L_{j l}\right)}$ is a survival probability of deteriorating product $l$. The quantity surviving of deteriorating product $l$ at the end of the time interval follows a binomial distribution with parameters $n=N_{l}$ and $p=e^{-\lambda_{l}\left(t_{j}+t_{i j} y_{i j l}+L_{j l}\right)}$. Hence,
$N_{l} e^{-\lambda_{l}\left(t_{j}+t_{i j} y_{i j l}+L_{j l}\right)}$ express the expected surviving quantity. The objective of the model is to minimize total expected costs of location, inventory, transportation, and loss of deteriorating product value. The first objective term $f_{j} x_{j}$ is fixed cost of DC located at retailer site $j$ per unit time. The second objective term is the sum of transportation costs, inventory costs, and the loss of deteriorating product value. Constraint (2) defines the capacity of each DC. Constraint (3) ensures that each deteriorating product can be delivered in usable lifetime period. Constraint (4) ensures that each retailer with demand for deteriorating product is supplied only by one DC. Constraint (5) illustrates that DC can provide service for retailers only when potential DC becomes a real DC. Constraint (6) defines the range of the reorder number of deteriorating product $l$ at DC $j$.

By introducing auxiliary variables $t_{j l}, z_{j l}$ for each $j$ and $l, t_{j l}$ is defined by constraint (14), and $z_{j l}$ is defined by constraint (15). Now we, based on the above formulation, give conic quadratic mixed-integer programming model (CQMIP):

$$
\min \sum_{j \in J}\left(f_{j} x_{j}+\sum_{l \in L}\left(\sum_{i \in I} \hat{d}_{i j l} y_{i j l}+\theta h_{l} \frac{z_{j l}}{2}+\hat{q}_{j l} t_{j l}+\sum_{i \in I} N_{i 0}\left(1-e^{-\lambda_{l}\left(t_{j}+t_{i l} y_{i j l}+L_{j l}\right)}\right)\left(F_{i}+D_{i}\right)\right)\right)
$$




$$
\begin{array}{ll}
\text { s.t. } & \sum_{l \in L}\left(L_{j l} \sum_{i \in I} \mu_{i l} y_{i j l}+Q_{j l}+t_{j l}\right) \leq C_{j} x_{j}, \quad j \in J, \\
& \left(t_{j}+L_{j l}+y_{i j l} t_{i j}\right) \leq t_{l}, \quad i \in I, \quad j \in J, \quad l \in L, \\
& \sum_{j \in J} y_{i j l}=1, \quad i \in I, \quad l \in L, \\
& y_{i j l} \leq x_{j}, \quad i \in I, \quad j \in J, \quad l \in L, \\
& Q_{j l} \geq 0, \quad j \in J, \quad l \in L, \\
& \sqrt{y_{. j l}^{\prime}\left(L_{j l} V_{l}+\sigma_{L_{j l}}^{2} M_{l}\right) y_{. j l} \leq t_{j l}, \quad j \in J, \quad l \in L,} \\
& \sum_{i \in I} H_{j l} \mu_{i} y_{i j l}^{2}+\left(Q_{j l}-\frac{z_{j l}}{2}\right)^{2}-\frac{z_{j l}^{2}}{4} \leq 0, \quad j \in J, l \in L, \\
& t_{j l}, z_{j l} \geq 0, \quad j \in J, l \in L,
\end{array}
$$

where $H_{j l}=\hat{F}_{j l} /\left(\theta h_{l} / 2\right)$.

Variable $t_{j l}$ is used to substitute the term $\sqrt{y_{. j l}^{\prime}\left(L_{j l} V_{l}+\sigma_{L_{j l}}^{2} M_{l}\right) y_{. j l}}$, and we can prove that problem $(P)$ is equivalent to CQMIP.

4.3. Extended Cover Cuts. We use optimization software CPLEX to solve CQMIP problem. In order to get a better solution we strengthen the formulations (CQMIP) with cover inequalities, utilize the left side of inequality (14) to substitute $t_{j l}$, and then we receive the nonlinear $0-1$ constraint knapsack result:

$$
\sum_{l \in L}\left(\sqrt{y_{. j l}^{\prime}\left(L_{j l} V_{l}+\sigma_{L_{j l}}^{2} M_{l}\right) y_{. j l}}+L_{j l} \sum_{i \in I} \mu_{i l} y_{i j l}\right) \leq c_{j} .
$$

For inequality (17), define a set function $f(s)$ that is from set $2^{I}$ to set $R$ :

$$
f(s)=\sum_{l \in L}\left(\sqrt{y_{. j l}^{\prime}\left(L_{j l} V_{l}+\sigma_{L_{j l}}^{2} M_{l}\right) y_{. j l}}+L_{j l} \mu(s)\right) .
$$

Using submodularity of set function $f(s)$, we can gain the extended cover cuts for the submodular knapsack set $Y$ :

$$
\begin{aligned}
Y & =\left\{y \in\{0,1\}^{|I|}: f(y) \leq c\right\}=\left\{y \in\{0,1\}^{|I|}:\right. \\
& \sum_{l \in L}\left(\sqrt{y_{. j l}^{\prime}\left(L_{j l} V_{l}+\sigma_{L_{j l}}^{2} M_{l}\right) y_{. j l}}+L_{j l} \sum_{i \in I} \mu_{i l} y_{i j l}\right) \\
& \leq c\} .
\end{aligned}
$$

Because the validity of the cover inequality depends on not only the submodular knapsack set $Y$ but also the cover set, define a cover set as follow: $S \subseteq I$, which is called a cover set for $Y$ if $\sum_{l \in L}\left(\sqrt{y_{. j l}^{\prime}\left(L_{j l} V_{l}+\sigma_{L_{j l}}^{2} M_{l}\right) y_{. j l}}+L_{j l} \mu(s)\right)>c x$.

Narayanan [27] shows that for cover set $S$, cover inequality $\sum_{i \in S} y_{i} \leq|s|-1$ is valid for $Y$. We will utilize this cover inequality in our computations.

\section{Numerical Experiments and Sensitivity Analysis}

In this section, we make numerical analysis to computational results about CQMIP formulations of the combined locationinventory problems in the deteriorating products supply chain and compare our computational results with the earlier Lagrangian relaxation method.

There are three data sets in our experiment: 18-node, 80 node, and 150-node date set. In Table 1, we give the results obtained by running CQMIP, compared with Lagrangian relaxation method. The results indicate that our method performs quite well in this experiment, reaching optimum solution faster than Lagrangian relaxation method.

In our method, we add extended cover inequalities; in Table 2, we give the results obtained by adding extended cover inequalities. We can find that, with capacity decrease, the problem becomes more difficult, but, adding extended cover inequalities, we can find that the solution time is reduced and the number of nodes is also reduced.

Now we examine the influence of correlated retailers' stochastic demands on the combined location-inventory problems in the deteriorating products supply chain. We utilize 18-node data set to illustrate the effect of retailers' stochastic demand correlation. In Figure 2, the retailers' demands are uncorrelated, and the links show retailer assignments in the optimal solution. Four DCs are opened in Beijing, Shanghai, Guangzhou, and Chengdu, and the total expected cost is 180090 . Now we add correlation to the 
TABLE 1: Comparing CQMIP and Lagrangian relaxation.

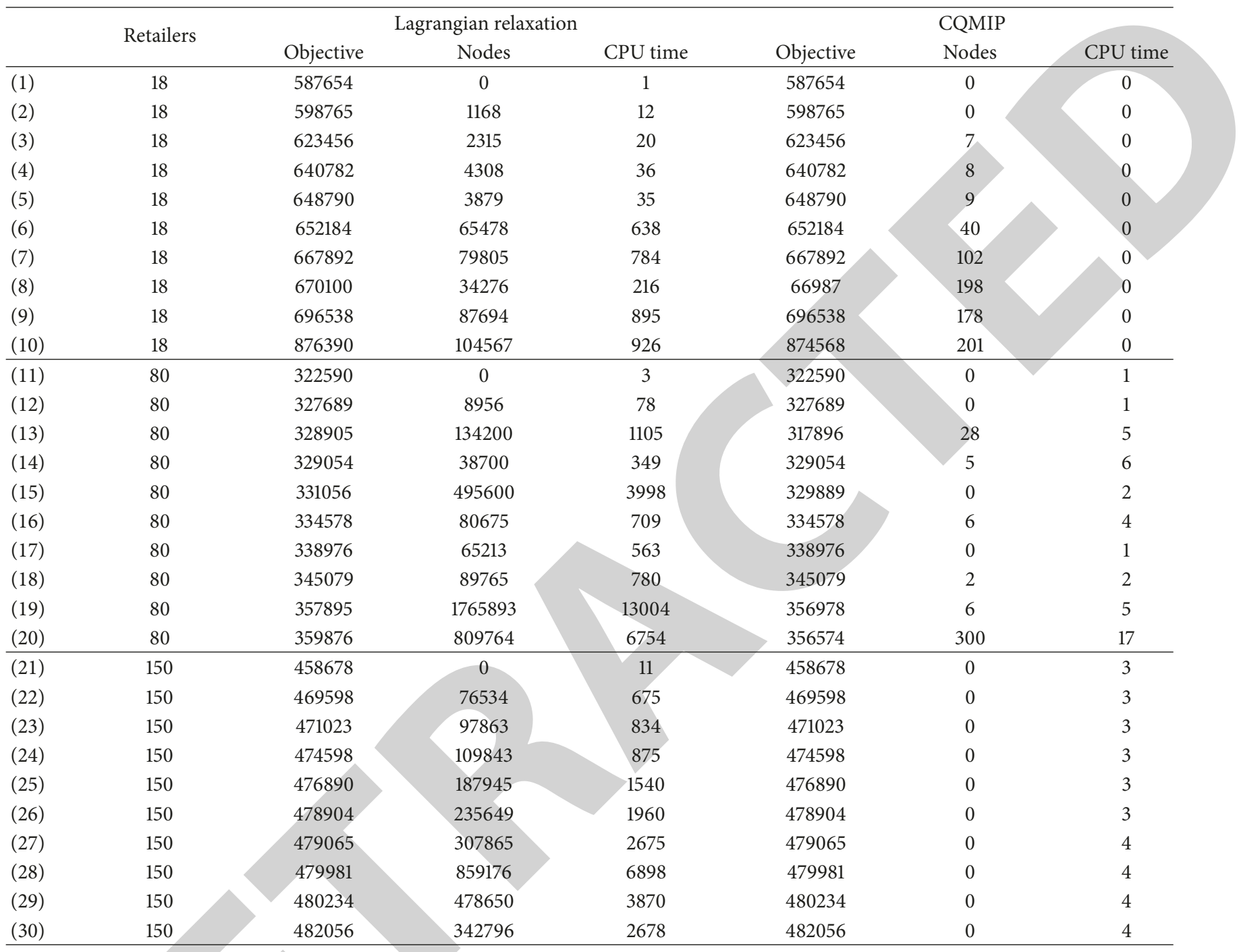

TABLE 2: The influence of capacity on solving CQMIP.

\begin{tabular}{|c|c|c|c|c|c|}
\hline \multirow[b]{2}{*}{$\begin{array}{l}\text { DC capacity } \\
\text { (\% demand) }\end{array}$} & \multicolumn{2}{|c|}{ PLEX } & \multicolumn{3}{|c|}{ CPLEX + cuts } \\
\hline & Nodes & CPU time & Nodes & CPU time & $\begin{array}{c}\text { Cover cuts } \\
\text { (extended cover cuts) }\end{array}$ \\
\hline 19.5 & 4090 & 70 & 3160 & 83 & $1573(0)$ \\
\hline 19 & 24316 & 324 & 6578 & 208 & $656(0)$ \\
\hline 18.5 & 10235 & 208 & 9878 & 179 & $589(0)$ \\
\hline 18 & 11934 & 206 & 7865 & 180 & $548(0)$ \\
\hline 17.5 & 27867 & 406 & 9654 & 369 & $497(0)$ \\
\hline 16.5 & 2898 & 78 & 5430 & 67 & $572(0)$ \\
\hline 16.3 & 7865 & 150 & 5587 & 106 & $576(0)$ \\
\hline 16.29 & 167543 & 2056 & 32789 & 784 & $579(0)$ \\
\hline
\end{tabular}

demands of the retailers, to see how correlation will change the solution. We assume the correlation between retailers is up to $80 \%$; in this case the total expected cost is 186595 . The current solution is no longer optimal, but the inventory level and subsequent costs are lower.
We consider positive correlation between retailers; Figure 3 shows the total expected cost is a function of the retailers' demands correlation; the total expected cost increases monotonically. Moreover, Figure 3 shows the number of opened DCs is a function of the retailers' 


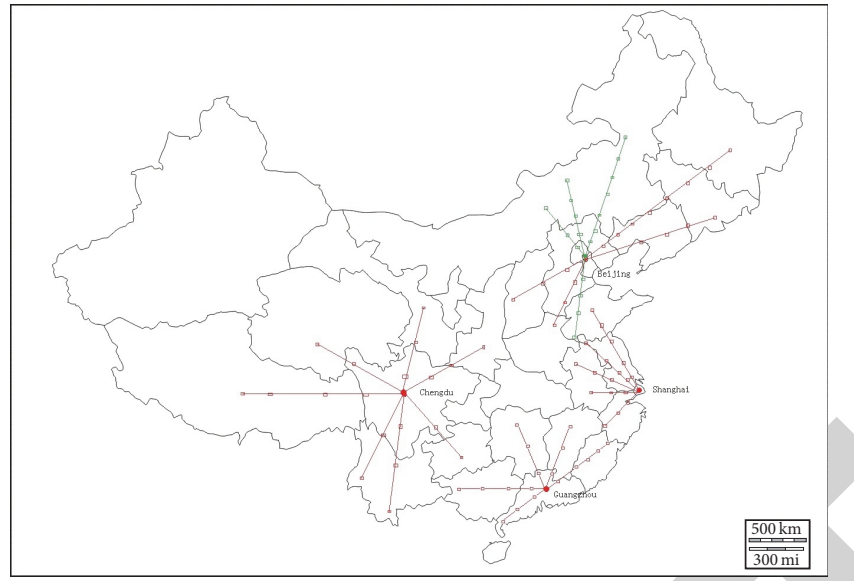

FIgURE 2: The effect of correlated retailers' demand on the supply chain.

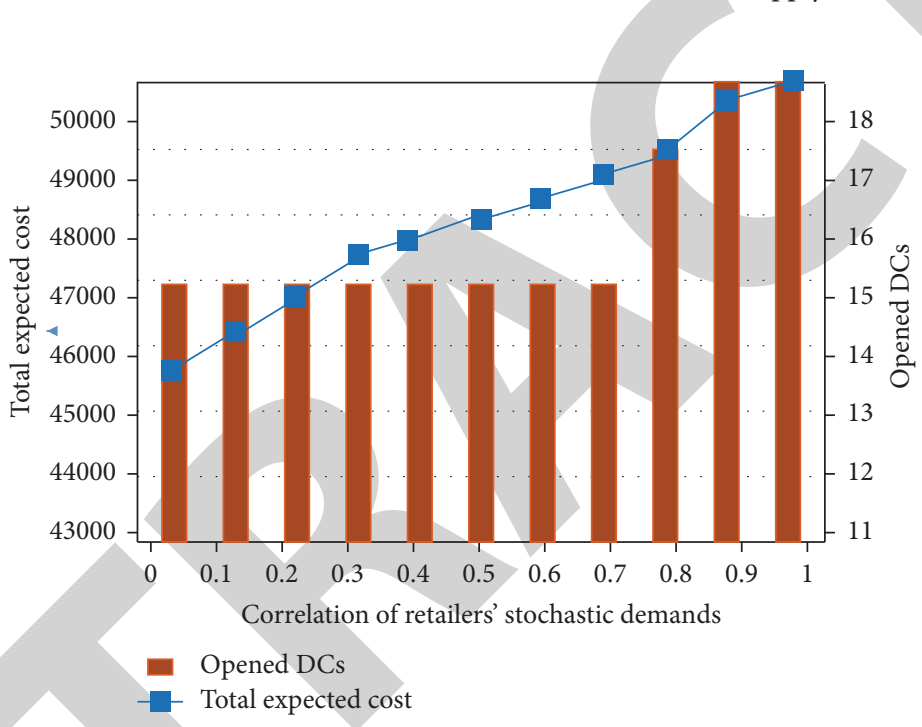

FIgURE 3: The total expected cost and the number of opened DCs are all function of the retailer stochastic demands correlation.

demands correlation; from this we can conclude that the retailers positive demands correlation reduces the risk of location.

In our model we consider lead time is stochastic; now let the standard deviation of the lead time be 0 ; Figure 4 shows this particular case. Based on Figure 4, in order to explain lead time variability, we add a third axis. The influence of retailers' correlation demands and stochastic lead times on total cost is captured by Figure 4; we can find that when retailers' stochastic demands are correlated and/or stochastic lead time standard deviation increases, the total cost of supply chain will be increased.

Now let the number of opened DCs increase to 18, but keep lead time standard deviation invariability; from Figure 5 we can conclude that with the retailers' stochastic demands correlation increase, there is an increase in the number of opened DCs. We also conclude that the number of opened DCs is a function of retailers' stochastic demands correlation and lead time standard deviation.

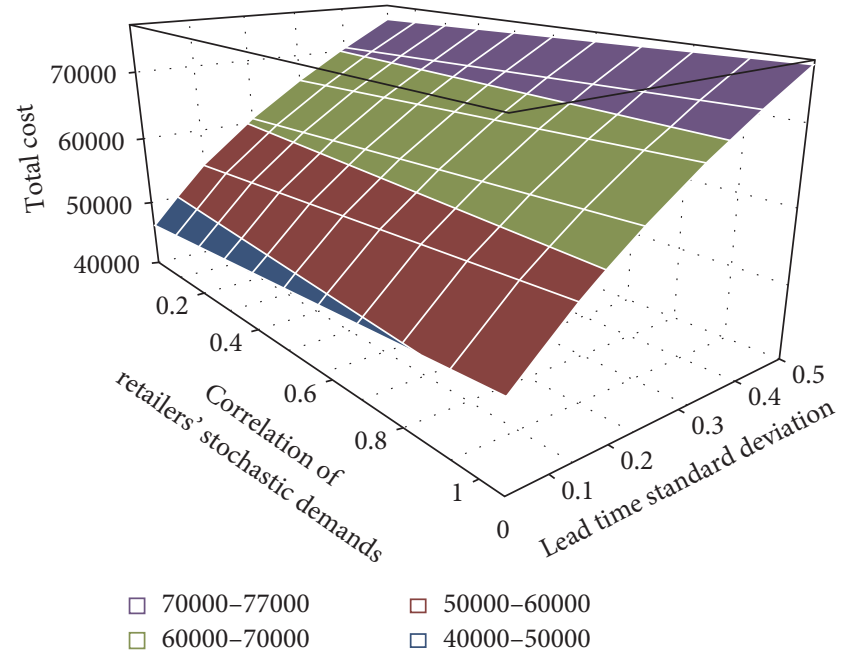

FIGURE 4: The total expected cost of the supply chain is a function of retailers' stochastic demands correlation and lead time standard deviation. 


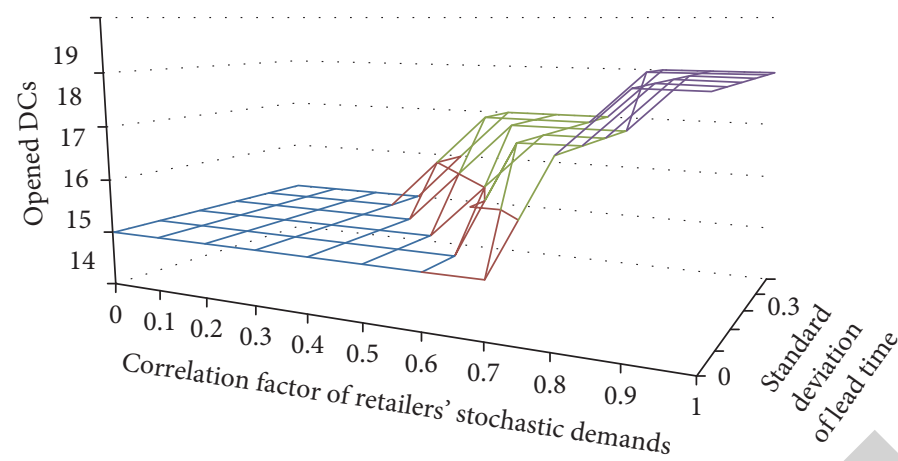

FIGURE 5: The number of opened DCs is a function of retailers' stochastic demands correlation and lead time standard deviation.
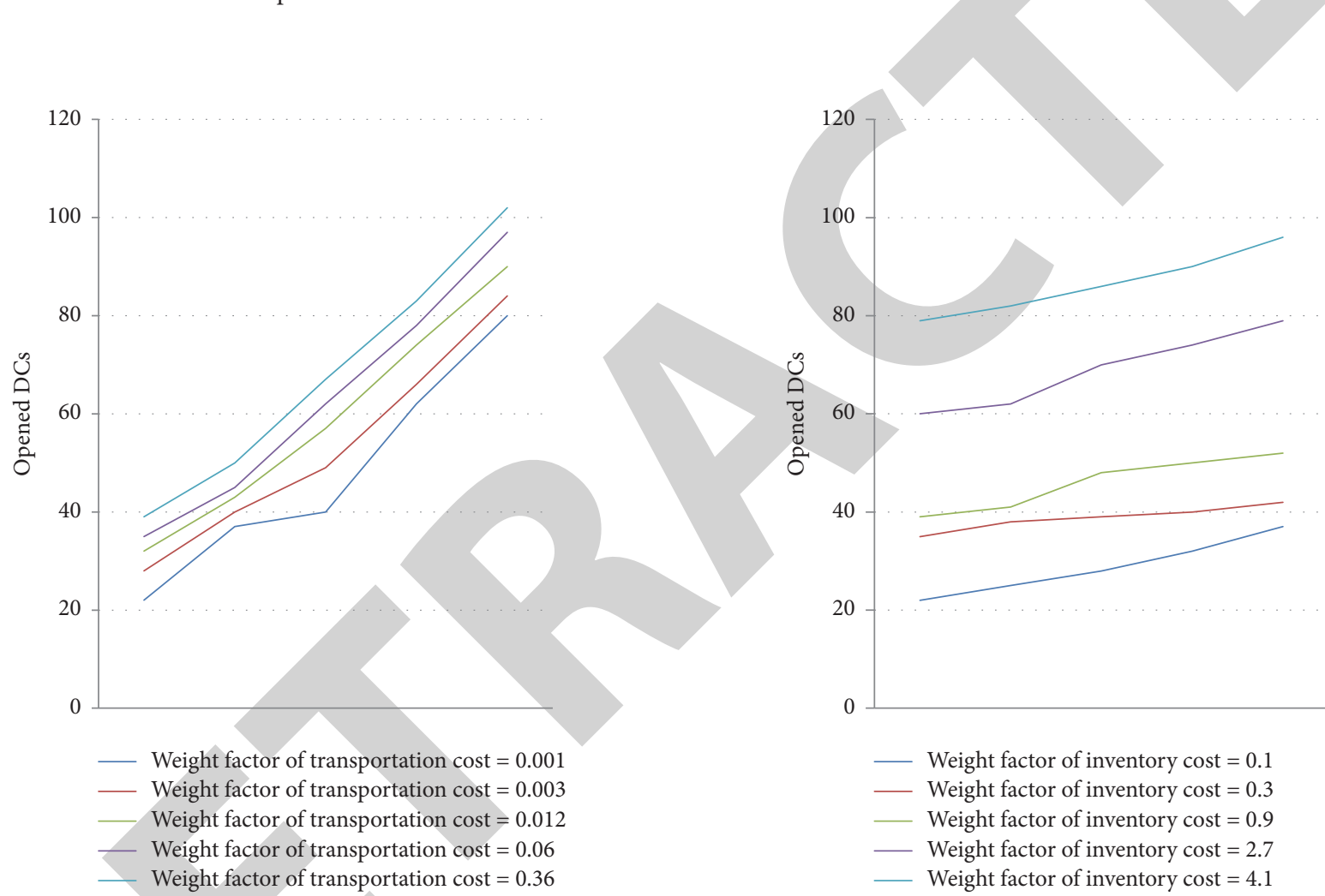

FIGURE 6: The number of opened distribution centers varies with the weight of transport cost $\beta_{l}$.

Now we examine the influence of $\beta_{l}$ and $\theta_{l}$ on the number of opened distribution centers. We utilize 150 -node data set to illustrate the influence; in our experiment the values of $\beta_{l}$ are $0.001,0.003,0.012,0.060$, and 0.360 , and the values of $\theta_{l}$ are $0.100,0.300,0.900,2.700$, and 4.100. From Figures 6 and 7 we can see that when the value of $\beta_{l}$ increases, the number of opened distribution centers increased, and when the value of $\theta_{l}$ increases, the number of opened distribution centers also increased. Because $\beta_{l}$ increases equally to the corresponding transportation cost increase, $\theta_{l}$ increases equally to the corresponding inventory cost increase, so the fixed-location cost has fewer impacts on the cost of the objective function; this results in more distribution centers being opened.

From Figures 8 and 9 we can see that when $\beta_{l}$ increases, the sum of total expected cost decreases, and when $\theta_{l}$
FIgURE 7: The number of opened distribution centers varies with the weight of inventory $\theta_{l}$.

increases, the sum of total expected cost increases. Figures 8 and 9 show the total expected cost as a function of $\beta_{l}$ and $\theta_{l}$. Taking into account inventory decision and location decision at the same time, it will bring a larger total expected cost reduction.

\section{Conclusions}

In this paper, we propose a new method to model and optimize combined location-inventory deteriorating products supply chain problem. We think capacity of facilities are limited, lead time is random, the products are varied, and retailers' random demands are correlated. These assumptions are more practical and near to actual supply chain circumstance. We use conic quadratic mixed-integer 


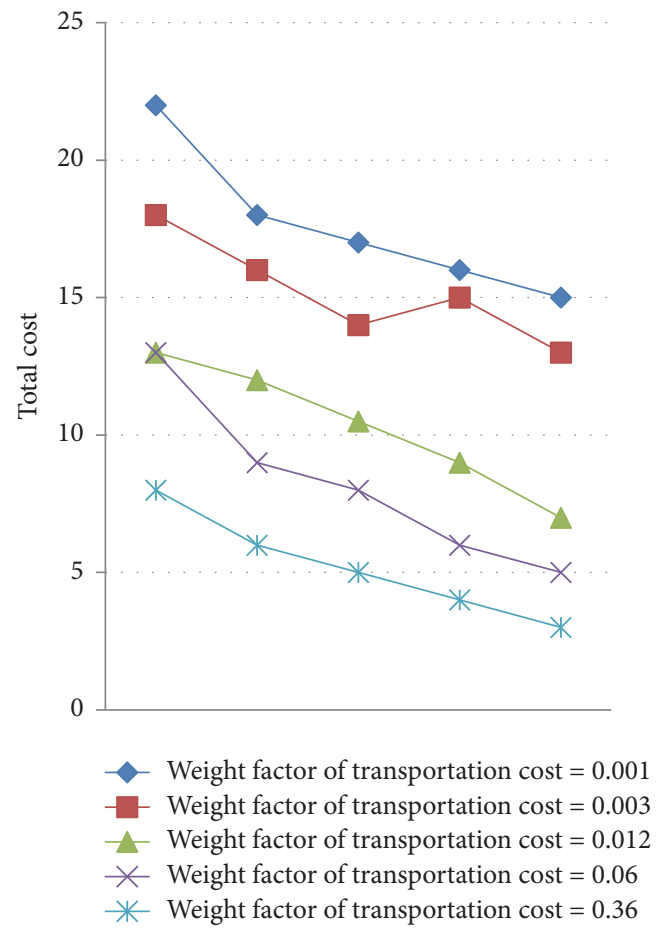

FIGURE 8: The total expected cost varies with the weight of transport $\operatorname{cost} \beta_{l}$.

programming to model integrated location and inventory problem of deteriorating products supply chain, and we used optimization software CPLEX to solve this problem. Not only does this new method obtain better computational solution than previous Lagrange methods, but also our model is useful to more general problems. Our method can be used for inventory management problems, location management problems, and even transportation management problems. Conic quadratic mixed-integer programming also can be used for service supply chain.

The research work provides an effective reference for managers of deteriorating products supply chain. Considering the correlation of the demand of the retailers, it can effectively reduce the inventory and reduce the cost of excessive inventory. It can better control the location of the distribution center and reduce the risks caused by improper location selection. With stochastic lead time standard deviation increase, the total cost of supply chain will increase. So supply chain managers need to control the stochastic lead time standard deviation in the right range. The number of opened DCs is a function of retailers' stochastic demands correlation and lead time standard deviation, so supply chain managers can effectively control the number of opened DCs in virtue of retailers' stochastic demands correlation and lead time standard deviation. The above measures make the supply chain achieve the minimum expected total cost.

In our model, we only consider combined locationinventory condition. In subsequent studies, we will continue studying combined location-inventory model with vehicle routing, applying conic integer programming approach to model combined production and transportation case.

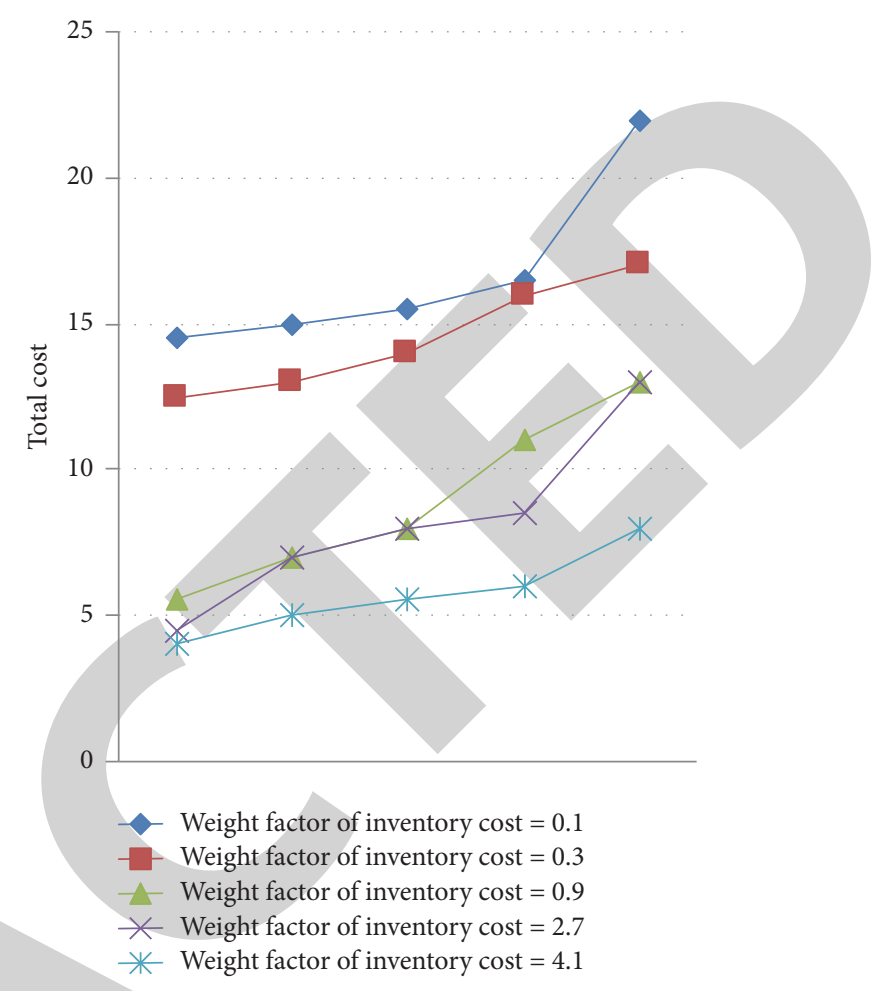

FIGURE 9: The total expected cost varies with the weight of inventory $\theta_{l}$.

\section{Conflicts of Interest}

The authors declare that they have no conflicts of interest.

\section{Acknowledgments}

This work is supported by Natural Science Foundation of China (no. 71640028, no. 71362030, no. 71262029) and Natural Science Foundation of Yunnan (no. 2013FZ048).

\section{References}

[1] K.-S. Wu, L.-Y. Ouyang, and C.-T. Yang, "An optimal replenishment policy for non-instantaneous deteriorating items with stock-dependent demand and partial backlogging," International Journal of Production Economics, vol. 101, no. 2, pp. 369384, 2006.

[2] S. K. Goyal and B. C. Giri, "Recent trends in modeling of deteriorating inventory," European Journal of Operational Research, vol. 134, no. 1, pp. 1-16, 2001.

[3] L. V. Snyder, M. S. Daskin, and C.-P. Teo, "The stochastic location model with risk pooling," European Journal of Operational Research, vol. 179, no. 3, pp. 1221-1238, 2007.

[4] Z. J. Shen and L. Qi, "Incorporating inventory and routing costs in strategic location models," European Journal of Operational Research, vol. 179, no. 2, pp. 372-389, 2007.

[5] L. Ozsen, C. R. Coullard, and M. S. Daskin, "Capacitated warehouse location model with risk pooling," Naval Research Logistics (NRL), vol. 55, no. 4, pp. 295-312, 2008. 
[6] Z.-J. M. Shen, C. R. Coullard, and M. S. Daskin, "A joint location-inventory model," Transportation Science, vol. 37, no. 1, pp. 40-55, 2003.

[7] M. S. Daskin, C. R. Coullard, and Z. . Shen, "An inventorylocation model: formulation, solution algorithm and computational results," Annals of Operations Research, vol. 110, pp. 83106, 2002.

[8] Z.-J. M. Shen and M. S. Daskin, "Trade-offs between customer service and cost in integrated supply chain design," Manufacturing and Service Operations Management, vol. 7, no. 3, pp. 188207, 2005.

[9] P. A. Miranda and R. A. Garrido, "Incorporating inventory control decisions into a strategic distribution network design model with stochastic demand," Transportation Research Part E: Logistics and Transportation Review, vol. 40, no. 3, pp. 183-207, 2004.

[10] S. J. Erlebacher and R. D. Meller, "The interaction of location and inventory in designing distribution systems," IIE Transactions, vol. 32, no. 2, pp. 155-166, 2000.

[11] A. Dasci and V. Verter, "A continuous model for productiondistribution system design," European Journal of Operational Research, vol. 129, no. 2, pp. 287-298, 2001.

[12] J. Asl-Najafi, B. Zahiri, A. Bozorgi-Amiri, and A. TaheriMoghaddam, "A dynamic closed-loop location-inventory problem under disruption risk," Computers \& Industrial Engineering, vol. 90, pp. 414-428, 2015.

[13] Z.-H. Zhang and A. Unnikrishnan, "A coordinated locationinventory problem in closed-loop supply chain," Transportation Research Part B: Methodological, vol. 89, pp. 127-148, 2016.

[14] A. Diabat, E. Dehghani, and A. Jabbarzadeh, "Incorporating location and inventory decisions into a supply chain design problem with uncertain demands and lead times," Journal of Manufacturing Systems, vol. 43, pp. 139-149, 2017.

[15] B. Vahdani, M. Soltani, M. Yazdani, and S. Meysam Mousavi, "A three level joint location-inventory problem with correlated demand, shortages and periodic review system: Robust metaheuristics," Computers \& Industrial Engineering, vol. 109, no. 7, pp. 113-129, 2017.

[16] N. Rich and P. Hines, "Supply-chain management and timebased competition: The role of the supplier association," International Journal of Physical Distribution \& Logistics Management, vol. 27, no. 3-4, pp. 210-225, 1997.

[17] V. D. R. Guide Jr., G. C. Souza, L. N. van Wassenhove, and J. D. Blackburn, "Time value of commercial product returns," Management Science, vol. 52, no. 8, pp. 1200-1214, 2006.

[18] J. Blackburn and G. Scudder, "Supply chain strategies for perishable products: the case of fresh produce," Production Engineering Research and Development, vol. 18, no. 2, pp. 129137, 2009.

[19] K.-J. Wang, Y. S. Lin, and J. C. P. Yu, “Optimizing inventory policy for products with time-sensitive deteriorating rates in a multi-echelon supply chain," International Journal of Production Economics, vol. 130, no. 1, pp. 66-76, 2011.

[20] B. C. Arntzen, G. G. Brown, T. P. Harrison, and L. L. Trafton, "Global Supply Chain Management at Digital Equipment Corporation," Interfaces, vol. 25, no. 1, pp. 69-93, 1995.

[21] S. Nahmias, "Perishable inventory theory: a review," Operations Research, vol. 30, no. 4, pp. 680-708, 1982.

[22] M. Yu and A. Nagurney, "Competitive food supply chain networks with application to fresh produce," European Journal of Operational Research, vol. 224, no. 2, pp. 273-282, 2013.
[23] L. M. M. Tijskens and J. J. Polderdijk, "A generic model for keeping quality of vegetable produce during storage and distribution," Agricultural Systems, vol. 51, no. 4, pp. 431-452, 1996.

[24] R. Akkerman, P. Farahani, and M. Grunow, "Quality, safety and sustainability in food distribution: A review of quantitative operations management approaches and challenges," OR Spectrum, vol. 32, no. 4, pp. 863-904, 2010.

[25] A. Ben-Tal and A. Nemirovski, Lectures on Modern Convex Optimization: Analysis, Algorithms, and Engineering Applications, MOS-SIAM Series on Optimization, Society for Industrial and Applied Mathematics (SIAM), Pennsylvania, Pa, USA, 2001.

[26] F. Alizadeh and D. Goldfarb, "Second-order cone programming," Mathematical Programming, vol. 95, no. 1, pp. 3-51, 2003.

[27] V. Narayanan, Lifting for Conic Mixed-Integer Programming, Springer-Verlag Inc, New York, NY, USA, 2011. 